BINARY NUCLEI OF PLANETARY NEBULAE

Agnès Acker

Observatoire de Strasbourg, France

In view of the spectral similarity and the probable evolutionary relation between certain planetary nebulae (PN) and other objects that are generally binaries (symbiotic stars, Be stars, or slow novae), it is probable that many nuclei of PN are double.

Astrometric observations (Cudworth, 1973) showed that six nuclei are binary. Also, 13 PN have a visible central star that is probably accompanied by a hotter star. If one counts other nuclei whose radial velocity and luminosity vary greatly, there are 36 nuclei in all that are probably double.

For seven of these nuclei, I took a series of spectra, in May and August, 1975, using two spectrographs with built-in image-tubes (77 and $90 \AA / \mathrm{mm}$, respectively). In three cases (Fg Sge, $\mathrm{BD}+66^{\circ} 1066$, and $\mathrm{BD}+30^{\circ} 623$, nuclei of $60-7^{\circ} 1,96+29^{\circ} 1$, and $165-15^{\circ} 1$, respectively), the radial velocity definitely varied, and for these a provisional period has been calculated.

ON THE VARIABILITY OF THE PLANETARY NEBULAE

\title{
E.B. Kostyakova
}

Sternberg State Astronomical Institute, U.S.S.R.

The study of several planetary nebulae, suspected earlier of variability, was continued during the last years at the Sternberg Astronomical Institute.

The photoelectric observations, carried out in 1968-1975 in Crimea and at the observatory Skalnate Pleso (Czechoslovakia), were examined together with the objective-prism spectrograms, obtained in a homogeneous way during several observing seasons in 1972-1975 with the 50-cm Maksutov telescope of the Crimean station.

The study confirmed the earlier conclusion ([1], and others) on the variability of planetaries NGC 6572 , 6891, IC 4997, and Hu2-1. The mentioned planetaries showed systematic changes of brightness in the range $0 m_{2}$ - $0 m 3$, both in photoelectric observations and in the spectral lines. 